\title{
Pilot study: An on-demand reminder system increases adherence of birth control pill users
}

\author{
Janne Sahlman ${ }^{1}$, Heikki Matero ${ }^{2}$ and Henna Kärkkäinen ${ }^{3 *}$ \\ ${ }^{1}$ Pohjola Hospital, Kuopio, Finland \\ ${ }^{2}$ Farenta Oy, Kuopio, Finland \\ ${ }^{3}$ Department of Gynegology and Obstetrics, Kuopio University Hospital, Finland
}

\begin{abstract}
Purpose of the article: In this study, we studied the feasibility of an on-demand reminder system to increase patient adherence to oral birth control pills. Additionally, we studied the impact of such a reminder solution for improving the consistency in pill-taking timing.

Materials and methods: 24 voluntary women using oral birth control pills participated in the study. An electronic device attached to the push-through pill packages monitored pill-taking for two months. During the first month the device merely monitored pill consumption behaviour, without interacting with the patient. During the second month of the study, a smartphone application connected to the device was activated and alerted the user only if she was about to forget to take the daily pill. Pill-taking time points were gathered to a cloud service and analysed.

Results: Without the alert system, 46\% (11 women) forgot to take one or more pills during the first one-month period. When the on-demand reminder system was turned on during the second month, $8 \%$ ( 2 women) of the women forgot one or more pills. The risk to forget the daily pill was reduced to one seventh with the ondemand reminder $(\mathrm{p}<0.01)$. The time window during which the participants took their pill decreased by 1 hour and 32 minutes ( $<<0.01)$. All participants completed the pilot, drop off rate being $0 \%$.
\end{abstract}

Conclusions: An on-demand reminder system seems to be well tolerated and can increase adherence to medication. An on-demand alert system may prove to be more useful in a long-term use than typical daily alerts.

\section{Introduction}

Poor patient adherence is a major problem in medical drug use and in clinical drug trials. It has been estimated that $50 \%$ of the patients use prescribed medical drugs as they were supposed to [1].

Oral contraception pills are a special group of medical drugs since the users are motivated and understand the importance of the pills. However, during the first year of oral contraceptive usage, nine percent of the users get pregnant because of poor adherence [2]. Forgetting from one to three pills in a cycle is a frequent problem concerning 15$51 \%$ of oral contraceptive users. This is thought to be due to young age, inability to establish a routine, pill unavailability, side-effects, loss of motivation and lack of involvement in the initial decision to begin oral contraception [3].

In 2015 Cochrane analysis of different methods to increase the adherence to oral contraceptives could not found any studies where simple daily text message reminders would increase adherence to contraceptive pills [4]. However, the daily text message reminders increase adherence to chronic disease medication from 50 to $67.8 \%$ [5]. However, the median duration of the studies in Thakkar's review was only three months. Thus, it remains to be shown if longer duration studies will show alert fatigue for the constant daily reminders and decrease of adherence improvement. In clinical support systems, constant repeated alerts have been shown to cause clinicians to override the alerts [6].

Electronic surveillance systems and on-demand text message alerts have been shown to improve the precision with which patients follow their prescription of oral antidiabetics in a six-month study period [7]. Surprisingly, the system could not decrease the number of missed doses.

Patient reporting on their adherence has been shown to be of low accuracy [8]. The electronic medication packaging devices provide accurate information of the time and date of the removal of the medication from the pill package. However, the high cost of the devices and their bulkiness have restricted their use in large population studies [9].

In this study, we studied the feasibility of a novel on-demand reminder system which consists of an electronic device (Figure 1) attached to a push-through pill package and a smartphone application in patients using oral contraceptives. Base line adherence was measured by using the on-demand reminder system without the reminders.

\section{Materials and methods}

\section{Study group}

The 24 women recruited for our study were over 18-years-old and had been using oral contraception pills. They volunteered for the

Correspondence to: Henna Kärkkäinen, Department of Gynecology and Obstetrics, Kuopio University Hospital, Puijonlaaksontie 2, P.O. Box 1777, 70211 Kuopio, Finland, E-mail: henna.karkkainen@kuh.fi

Key words: birth control, contraceptive methods, patient adherence, smartphone application, reminder system

Received: March 20, 2018; Accepted: March 29, 2018; Published: April 03, 2018 


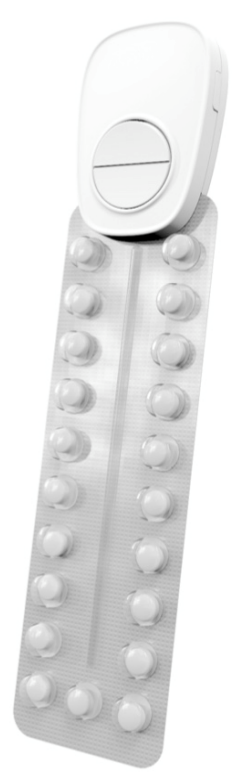

Figure 1. The device monitoring the pills taken from the push-through pilll package (Popit Inc).

study and did not receive any financial benefit of the study. The ethical committee of the Kuopio University approved the study protocol.

\section{On-demand reminder system}

The on-demand reminder system consisted of an electronic device which was attached to a push-through pill package, a smartphone application, and a cloud service to store recorded data (Popit Ltd., 2017).

The electronic device detected the pill-taking from a push-through pill package and recorded the extraction time. The electronic device used sensors for detecting motion of the device, touching the pushthrough pill package and mechanical vibration of the push-through pill package caused by breaking of the aluminium foil when extracting a pill. This information was combined with the pill removal events. Motion and touch sensors were used for filtering out false positive detections caused by other vibration than actually taking the pill out of the push-through pill package.

Information about detected pill removals was delivered to the smartphone application, which provided the on-demand alert for the user if she was about to forget to take a pill. Also, the application showed history information in a form of a pill diary. The smartphone application could be configured into a locked mode, where on-demand reminders and pill diary were not available but all information from the electronic device was still collected. This mode was used during the first month when the patient's adherence baseline was determined.

All data received from the electronic device, as well as manual inputs from the smartphone application user interface, were delivered to the cloud service, where the data was stored anonymously. Data analysis was performed using the data stored in the cloud service.

\section{First interview}

At the first interview the study protocol was explained to the participants and written consent was received. Then, the electronic device was attached to the oral contraception push-through pill package of the participant and the application was downloaded onto their smartphone. Also, the participant was shown how to change the electronic device to a new push-through pill package in case it needed to be changed.

\section{Phase one - silent surveillance}

During the first month of the study, the electronic device was used to register the time points at which pills were taken. The smartphone application was locked, and the user could not use it. During this phase, the smartphone application did not provide on-demand alerts.

\section{Phase two with alert turned on}

After one-month surveillance a researcher met the participants and turned the smartphone application's on-demand reminder on and unlocked the smartphone application. During this phase, the smartphone application's on-demand reminder system alerted the participant if she was about to forget the pill. Smartphone application was programmed to alert one hour after the time point the participant had set herself, if she had not taken the pill before that time. After phase two, the electronic device was collected and the two-month surveillance data was checked with user to be accurate.

\section{Statistical analysis}

Non-adherence was determined as failing to take the pill once or more in one-month surveillance period. The mean and standard error of mean (SEM) is expressed for forgotten pills per person.

Results for pill-taking regularity is expressed as a mean difference from 24-hour cycle and standard deviation (SD) is used for variance. The significance of the differences is evaluated using one-tailed paired Student $\mathrm{T}$-test. The data was visually estimated to be normally distributed. The difference in the risk for forgetting was evaluated using risk ratio.

\section{Results}

\section{Forgetting the pill}

During the first month without the on-demand reminder system there were on average $0.58+/-0,63$ (SEM) (1.9\%) missed pills per user (Table 1). During the second month there were $0.08+/-0,15$ (SEM) $(0.3 \%)$ missed pills $(\mathrm{p}<0.01)$. Risk ratio for forgetting pills with or without the on-demand reminder system was $14.3 .45 .8 \%$ of the users forgot one or more pills during the first month and $8.3 \%$ during the second month.

\section{Pill taking regularity}

The mean difference from daily 24-hour cycle was 3 hours and 54 minutes $+/-2$ hours and 5 minutes (SD) during the first month and 2 hours and 22 minutes $+/-1$ hour and 24 minutes (SD) during the second month - the difference being 1 hour and 32 minutes $(\mathrm{p}<0.01)$ ). None of the participants stopped using the electronic device and smartphone application prematurely.

\section{Pregnancies}

None of the women in our trial got a positive pregnancy test during the study or in two months after the end or the study.

\section{Discussion}

The most important reason for non-intended pregnancies during oral birth-control is poor adherence. The pills are forgotten and taken irregularly [10]. In perfect use oral contraceptives are highly effective 
Table 1. Data showing number of users who forgot to take the pills.

\begin{tabular}{|c|c|c|}
\hline User code name & $\begin{array}{l}\text { Number of forgotten pills } \\
\text { without the reminder }\end{array}$ & $\begin{array}{l}\text { Number of forgotten pills } \\
\text { with the reminder }\end{array}$ \\
\hline cookie & 2 & 1 \\
\hline saki & 1 & 0 \\
\hline snuggles & 1 & 0 \\
\hline star & 0 & 0 \\
\hline pearl & 1 & 0 \\
\hline paws & 0 & 0 \\
\hline tweety & 0 & 0 \\
\hline pamela & 0 & 0 \\
\hline saju2 & 1 & 0 \\
\hline elis & 0 & 0 \\
\hline pitpit & 0 & 0 \\
\hline audrey & 0 & 0 \\
\hline rhoda & 0 & 0 \\
\hline cookie2 & 0 & 0 \\
\hline camill & 1 & 0 \\
\hline tillie & 0 & 0 \\
\hline elis2 & 0 & 0 \\
\hline pumpkin & 2 & 1 \\
\hline ambe & 1 & 0 \\
\hline tessie & 0 & 0 \\
\hline mario & 1 & 0 \\
\hline paws2 & 0 & 0 \\
\hline vinnie & 1 & 0 \\
\hline myr & 2 & 0 \\
\hline Mean & 0.58 & 0.083 \\
\hline Standard error of mean & 0.63 & 0.15 \\
\hline $\begin{array}{l}\text { Number of users with one } \\
\text { or more forgotten pills }\end{array}$ & 11 & 2 \\
\hline
\end{tabular}

but in typical use users report a $9 \%$ failure rate during the first year of use [2]. In a recent study, high adherence to oral contraceptives - defined one or less missed pills in a month - was estimated to be $54 \%$ based on patient reports and prescription claims [11]. Similarly, during the first phase of our study, there were $46 \%$ of users who forgot one or more pills during the one-month surveillance without alerts. To help women not to forget the pill and improve adherence to birthcontrol medication, electronic reminder systems have been advocated. However, daily text messages have not been able to improve adherence to birth control pills [12].

During the second phase of our study the on-demand reminder system alerted if the user was about to forget the daily pill. Also, the user was able to check from the application, if the pill was already taken during the day. Hence, only 8 percent of the users forgot one or more pills during the one-month lasting second phase - the risk for forgetting dropped $86 \%$. In a recent review, in chronic disease medication constant daily reminders dropped risk for forgetting 33\% [5]. The reason for this difference may be the special nature of birthcontrol medication and also the patient group, which in our study was young women who were used to smartphone in their daily life.

The time window during which the participants took their pill narrowed by 1 hour and 32 minutes with the on-demand reminder turned on $(\mathrm{p}<0.01)$. Also, in Vervloet's work, an on-demand reminder system could help patients to take oral antidiabetics more accurately [7]. The decrease in the daily variation of pill taking time may have an important role especially in using the progestin-only pills, which lose their birth-control efficacy as the time variance increases to three or more hours.
Despite the short duration of our pilot study, it seems probable that an on-demand alert system is tolerated better than constant daily alerts. Accordingly, the drop-out rate of our study was zero. Also, the desensitization phenomenon for the on-demand reminder system is not likely, as the alert reminders are relatively rare.

The weaknesses of our study include the fact that the system was not capable of determining if the user actually ingested the pill which was monitored to been taken from the pill package. Also, the study followup time was quite short and the users' knowledge of the monitoring may have decreased the number of the forgotten pills both during the first and second phase of the study.

In our study, an on-demand reminder system could monitor the oral contraceptive pill taking accurately and forgetting the medication doses was significantly less frequent with the on-demand reminders. None of the patients in our study got pregnant during the trial, but it seems feasible that fewer forgotten contraceptive pills could lower the unintended pregnancy rate among the pill users. Also, regularity in birth control pill taking may lead to fewer unwanted side effects.

Hence, the results of this pilot study suggest that an on-demand reminder system can be used for measuring and increasing the adherence to medication. In the future, a longer follow up and a bigger study group would provide information of the adherence in a longterm surveillance. Also, the effect of the on-demand reminder system on the unintended pregnancies could be assessed.

\section{Acknowledgements}

The authors would like to thank the women who attended our study voluntarily for the invaluable help in our study.

\section{Funding of the study}

The on-demand reminder system for this study was obtained without cost from Popit Ltd.

\section{Competing Interests}

In accordance with our ethical obligation as researchers, we are reporting that Heikki Matero Ph.M. and Janne Sahlman M.D. Ph.D. own a small amount of stocks of the Popit Ltd., which provided the on-demand reminder system for our study. No salary or other financial support was obtained from the Popit Ltd. company during or after the study.

\section{References}

1. Haynes RB, McDonald H, Garg AX, Montague P (2002) Interventions for helping patients to follow prescriptions for medications. Cochrane Database Syst Rev 2: CD000011. [Crossref]

2. Kost K, Singh S, Vaughan B, Trussell J, Bankole A (2008) Estimates of contraceptive failure from the 2002 national survey of family growth. Contraception 77: 10-21. [Crossref]

3. Chabbert-Buffet N, Jamin C, Lete I, Lobo P, Nappi RE, et al. (2017) Missed pills: Frequency, reasons, consequences and solutions. Eur J Contracept Reprod Health Care 22: 165-169. [Crossref]

4. Smith C, Gold J, Ngo TD, Sumpter C, Free C (2015) Mobile phone-based interventions for improving contraception use. Cochrane Database Syst Rev 26: CD011159. [Crossref]

5. Thakkar J, Kurup R, Laba TL, Santo K, Thiagalingam A, et al. (2016) Mobile telephone text messaging for medication adherence in chronic disease: A meta-analysis. JAMA Intern Med 176: 340-9. [Crossref]

6. Ancker JS, Edwards A, Nosal S, Hauser D, Mauer E, et al. (2017) Effects of workload, work complexity, and repeated alerts on alert fatigue in a clinical decision support system. BMC Med Inform Decis Mak 17: 36. 
7. Vervloet M, van Dijk L, Santen-Reestman J, van Vlijmen B, van Wingerden P, et al. (2012) SMS reminders improve adherence to oral medication in type 2 diabetes patients who are real time electronically monitored. Int J Med Inform 81: 594-604. [Crossref]

8. Velligan DI, Wang M, Diamond P, Glahn DC, Castillo D, et al. (2007) Relationships among subjective and objective measures of adherence to oral antipsychotic medications. Psychiatr Serv 58: 1187-92. [Crossref]

9. Lam WY, Fresco P (2015) Medication adherence measures: An overview. Biomed Res Int 2015: 217047. [Crossref]
10. Halpern V, Lopez LM, Grimes DA, Stockton LL, Gallo MF (2013) Strategies to improve adherence and acceptability of hormonal methods of contraception. Cochrane Database Syst Rev 26: CD004317. [Crossref]

11. Nelson HN, Borrero S, Lehman E, Velott DL, Chuang CH (2017) Measuring oral contraceptive adherence using self-report versus pharmacy claims data. Contraception 96: 453-459. [Crossref]

12. Hou MY, Hurwitz S, Kavanagh E, Fortin J, Goldberg AB (2010) Using daily textmessage reminders to improve adherence with oral contraceptives: A randomized controlled trial. Obstet Gynecol 116: 633-640. [Crossref]

Copyright: $@ 2018$ Sahlman J. This is an open-access article distributed under the terms of the Creative Commons Attribution License, which permits unrestricted use, distribution, and reproduction in any medium, provided the original author and source are credited. 